\title{
ESTIMATION OF THE FEASIBILITY OF UNDERLAY INBAND DEVICE-TO-DEVICE COMMUNICATION BASED ON RADIO CONDITIONS IN LTE
}

\author{
Mohammad T. Kawser ${ }^{1 *}$, Md. Nazrul Islam Siddique ${ }^{2}$, Zunaed Kibria ${ }^{3}$ and \\ Abdullah Al Noman ${ }^{4}$ \\ ${ }^{1,2,3,4}$ Electrical and Electronic Engineering Department, Islamic University of \\ Technology, Bangladesh \\ 11mkawser@hotmail.com, ${ }^{2}$ nazruleee@iut-dhaka.edu, \\ ${ }^{3}$ zunaedkibria1992@gmail.com, ${ }^{4}$ shawon.noman@gmail.com
}

\begin{abstract}
Device-to-Device (D2D) communication, a feature of LTE-Advanced, allows the UEs to communicate with each other directly, rather than hopping through the eNodeB. It can offer many different advantages, including increased resource utilization. However, in underlay inband mode, D2D operation with limited interference level is a pivotal factor for the expected outcome. This restricts the feasibility of setting up a D2D communication unless proper radio conditions can be met. A prior estimation of such feasibility can help in optimized handling of the radio network. In this paper, we propose a novel but simple computation method, based on ensuring sufficiently high signal-to-noise ratio (SINR), to quickly estimate the feasibility of setting up underlay inband D2D communications in a cell. We also demonstrate sample computations using the proposed method.
\end{abstract}

Keywords - D2D communication, Underlay Inband Mode, ProSe, SINR

\section{INTRODUCTION}

Long term evolution (LTE), and its later versions LTE-Advanced, LTE-Advanced Pro, and $5 \mathrm{G}$ are the latest steps in the advancing series of cellular communication technologies. The Device-to-Device (D2D) communication or Proximity Service (ProSe), introduced in LTE-Advanced, has attracted the attention of researchers around the world, because of its various advantages. It is also considered as one of the promising techniques for the deployment of 5G. Due to the proximity of D2D UEs (DUEs), D2D can provide high throughput as well as low power consumption, leading to high energy efficiency [1], [2]. Moreover, since data are transferred directly between D2D users, they experience a much lower delay. In addition, the reuse of the same radio resources between the D2D users and the cellular users can increase the spectral efficiency [3]. D2D has many potential applications and it can be useful for multicasting [4], peer-to-peer communication [5], sharing video content or video dissemination [5]-[8], machine-to-machine (M2M) communication [9], gaming, traffic offloading [10], connectivity extension, and so forth [11].

In a cellular area, cellular UEs coexist with D2D UEs but their communication modalities differ from each other. In the case of the popular underlay inband mode of D2D communication, the same radio resources are shared by the D2D pair and a cellular user, for increased spectral efficiency. However, this communication mode introduces interference, potentially degrading the performance and adding complications in terms of 
interference control overhead and protocols. Thus, the D2D communication can only be set up when the interference is enough limited. In other words, the radio condition has to fulfill the requirements to allow setting up a D2D communication. A prior estimation of the number of possible D2D communications in a cell can be useful in both radio network planning (RNP) and radio network optimization (RNO). The authors of [12] analyzed the maximum allowable distance for the feasible D2D communication considering both uplink and downlink transmission while the multi-cell interference is taken into account. However, no research outcome directly indicates any suitable method for the estimation of the feasibility of setting up a D2D communication for a particular network condition. In this paper, a novel and quick method is proposed for such estimation. In the proposed method, the feasibility depends on how easily a suitable cellular user can be found, who can share resources with the D2D user, satisfying the necessary radio conditions. The proposal is explained only for the use of uplink resources of LTE FDD for D2D operation. This is because LTE FDD is more popularly used than LTE TDD and the spectrum is usually less occupied in uplink than that in downlink.

The remainder of the paper is outlined as follows. Section 2 discusses the management of radio resources for D2D communication. Section 3 depicts a system model for analysis and derives relevant relationships. Section 4 explains the proposed method for prior estimation of the feasibility of setting up D2D communications. Section 5 presents the simulation procedure and results. Finally, the whole paper is concluded in Section 6.

\section{RADIO RESOURCE MANAGEMENT FOR D2D COMMUNICATION}

D2D communication is a proximity-based service. D2D communication can be established for three different scenarios, namely, in coverage, out of coverage and partial coverage. In the coverage scenario, the eNodeB assigns specific resources to the transmitting UE and interferences with the cellular users can be avoided. In out of coverage scenario, the UE uses resources, which are preconfigured and there is no coverage on the frequency used for D2D communication. In the case of partial coverage scenario, the UE, in out of coverage, uses the pre-configured values, whereas the UE in coverage has its resources from the eNodeB and there should be careful coordination between the network and preconfigured values to limit the interferences at the UE.

Usually, D2D communication uses resources from uplink rather than downlink, primarily because the uplink spectrum is less occupied. The eNodeB can manage radio resources in three types of communication mode, such as, cellular mode, dedicated mode, and shared mode. The cellular mode is used for managing UEs, which transmit information via eNodeB. As D2D communication avoids hopping through eNodeB, dedicated mode and shared mode are used for resource management. The dedicated resources are assigned for D2D communication in the dedicated mode. On the contrary, in shared mode, cellular UEs and D2D UEs use the same resource pools. The classification of D2D communication, in terms of the spectrum assignment, is shown in Figure 1.

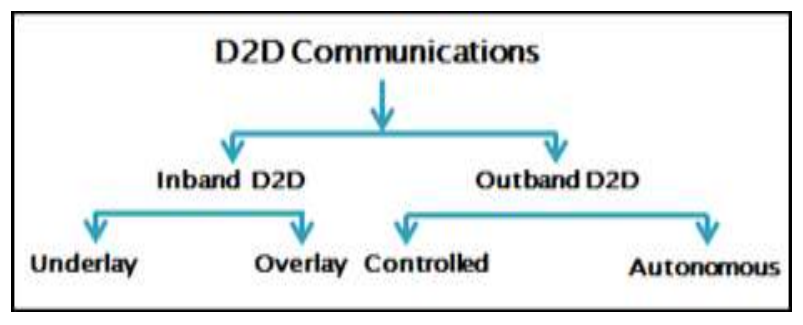

Fig. 1 D2D Classification 


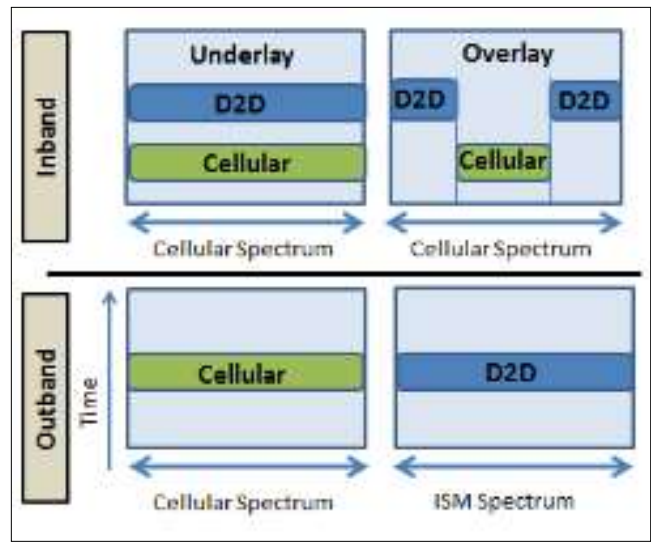

Fig. 2 Schematic representation of overlay inband, underlay inband and outband D2D

\subsection{INBAND MODE}

In order to exploit the licensed cellular band for D2D connection, sidelink communication uses resource pools (RP) assigned by the eNodeB. In inband D2D mode, it is easier to control spectrum usage, interference management, and network security, and it can provide a better quality of service (QoS). The inband mode is subdivided into underlay and overlay communication as shown in Figure 2. In underlay inband mode of D2D operation, the resource blocks (RBs), fed to D2D UEs by the eNodeB, are reused for cellular UEs. This increases the spectral efficiency but the overall performance can degrade due to interference on the shared resource blocks. Figure 3 depicts the interference condition with an example of two D2D pairs, pair 1 and pair 2, and a cellular user UE0. UE0 uses a set of RBs for uplink transmission, from which a part is used by pair 1 and the rest of the RBs are used by pair 2. As Figure 3 indicates, when a UE of D2D pair 2 receives signal from the other UE of the pair, the uplink transmission of UE0 causes significant interference. Similarly, when the eNodeB receives signal from UE0, the signal from a D2D pair can cause significant interference. For underlay inband mode, in order to enhance and strengthen the performance of different targets, such as, spectrum efficiency, energy efficiency, and cellular coverage, different approaches are proposed including diversity techniques, interference reduction, resource allocation and network coding [13] - [18].

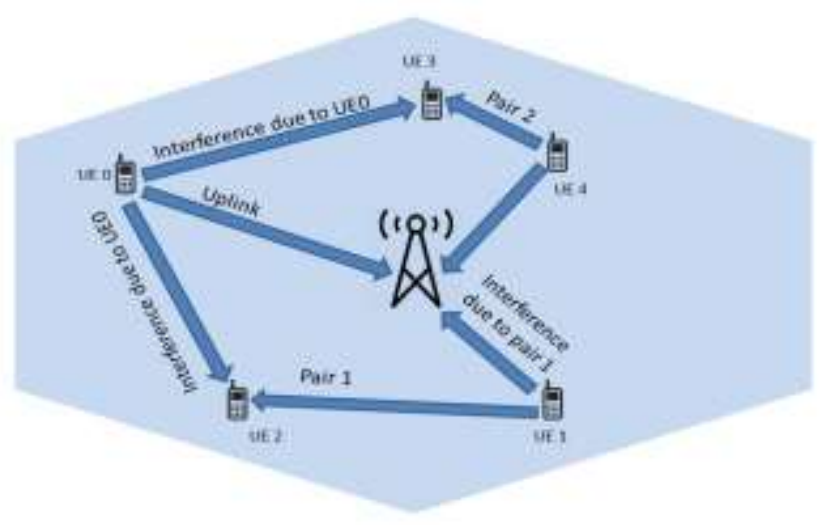

Fig. 3 Interference Condition with the Co-existence of D2D Pairs and a Cellular User, using the same Resources 
Unlike the underlay mode, dedicated resources are provided for the overlay inband mode of communication. This approach removes the difficulties of interference management but it reduces spectrum utilization and efficiency.

\subsection{OUTBAND MODE}

In outband D2D communication, the dedicated resource blocks are provided for D2D UEs, which can avoid interferences. Outband communication is subdivided into two types, namely controlled, when managed by the cellular network and autonomous, when it is operated on its own. ISM band is proposed for outband D2D communication. In ISM band, the channel contention between WLAN and D2D users may degrade the overall performance. To mitigate this problem, the authors of [19], [20] suggest forming clusters among the controlled D2D users, who are in Wi-Fi range. In this case, spectral efficiency, power efficiency, fairness, and data throughput increase. The authors of [21] propose caching the video files in cellular network and using D2D communication for viral videos.

\section{SYSTEM MODEL}

We consider the underlay inband mode of D2D operation with the same resource blocks shared by a D2D UE pair and a cellular UE. We assume 3 cells, each with $120^{\circ}$ angular separation, under an eNodeB. We assume a fixed position for the D2D pair, at the cell edge across the cell radius, with two-dimensionally variable positions in the cell for the cellular UE. Thus, as shown in Figure 4, the eNodeB, the D2D pair, and the cellular UE can be considered in a triangular position having specified distances. The shaded region indicates the coverage area of the cell, with distance $m+n$ as the cell radius. As the cellular UE changes its position, the SINR and hence, the data rate experience of the UEs will vary. Other distances, indicated in Fig. 4, can be expressed as

$$
\begin{aligned}
& x=\tan ^{-1} \frac{l}{m} \text { and } y=\tan ^{-1} \frac{l}{n} \\
& a=\frac{n}{\cos (y)} \\
& b=\frac{m}{\cos (x)}
\end{aligned}
$$

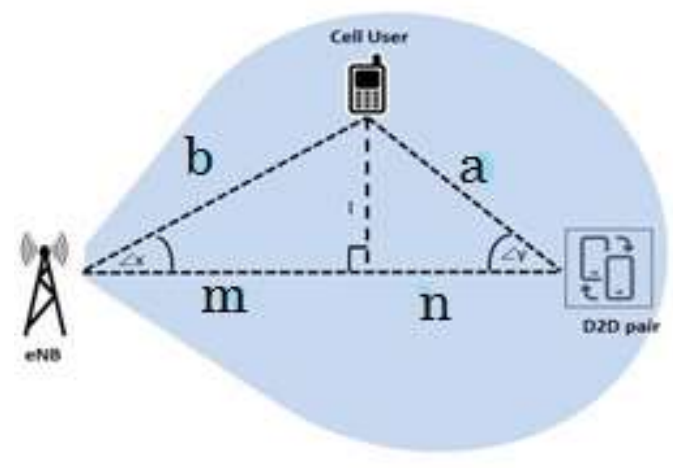

Fig. 4 Locations of the eNodeB, D2D and Cellular User in the Cell

\subsection{CHANNEL GAIN BETWEEN ENODEB AND CELLULAR UE}

Considering the channel gain dominantly affected by Rayleigh fading $\mathrm{H}$, log-normal shadowing $X_{\alpha}$, and path losses $-P L_{d B, B, u}$, the channel gain between eNodeB and a cellular user can be shown as [21] 
$G_{q}^{k}=10^{\left(-P L_{d B, B, u}+X_{\alpha}\right) / 10} \cdot\left|H_{k}\right|^{2}$

Here, the path loss, $P L_{d B, B, u}$ can be calculated using the equation [13],

$\left.P L_{d B, B, u}(d)=40\left(1-4 * 10^{-3}\right) h_{b}\right) \cdot \log _{10}\left(\frac{d}{1000}\right)-18 \log _{10}\left(h_{b}\right)-21 \log _{10}\left(f_{c}\right)+80$

where $f_{c}$ is the carrier frequency in $\mathrm{MHz}, h_{b}$ is the base station antenna height in meters, and $d$ is the distance between eNodeB and UE.

\subsection{CHANNEL GAIN BETWEEN THE USERS OF A D2D PAIR}

The two users in a D2D pair communicate over a direct path and short distance. The channel gain between the two users, $u$ and $v$, in the D2D pair, can be shown as [22]

$$
G_{u v}=K_{u v} d_{u v}^{-\alpha}
$$

where $\alpha$ is constant path loss exponent, $K_{u v}$ is normalized constant, and $d_{u v}$ is the distance between users, $u$ and $v$.

\subsection{UPLINK TRANSMIT POWER OF CELLULAR UE}

The uplink transmit power of a cellular user can be calculated using the expression of uplink power transmission, shown as [23]

$P_{U}=P_{O_{-} P U S C H}+\alpha_{1} \times P L+10 \log _{10} M_{P U S C H}+\Delta_{T F}+f_{T P C}$

where $P_{O_{-} P U S C H}$ is the basic parameter for power control and it is configured by the eNodeB. The UE estimates the path loss in downlink, $P L$ by measuring the linear average over the power contributions of the resource elements on cell specific reference signals within the considered frequency bandwidth and using the parameter, ReferenceSignalPower sent by the eNodeB. $\alpha_{l}$ allows the eNodeB to configure the degree to which the UE's estimate of the path loss in downlink would be taken into account in uplink power control. $\mathrm{M}_{\mathrm{PUSCH}}$ indicates the number of resource blocks (RBs) allocated for uplink transmission and it attempts to keep transmit power spectral density (PSD) constant. $\Delta_{\mathrm{TF}}$ allows the transmitted power per resource block to be adapted according to the level of MCS, and this compensation for MCS can optionally be deactivated, which sets $\Delta_{\mathrm{TF}}=0 . \mathrm{f}_{\mathrm{TPC}}$ is used for continuous closed-loop power adjustments and the eNodeB sends transmit power control (TPC) command to set the value of $\mathrm{f}_{\mathrm{TPC}}$. To determine the uplink transmit power using (7), $P L$ can be calculated using (5), with the distance $d$, shown as $b$ in Fig. 4.

\subsection{SINR EXPERIENCE}

The major source of interference for underlay inband mode of D2D communication is the sharing of uplink resources, as explained in Section 2. To estimate the impact of these interferences, the relevant SINR can be calculated. While a D2D user receives signal from its pair, it also receives interference from the uplink transmission of the cellular user using the same resources. Thus, the SINR of the D2D user can be expressed as

$\gamma_{\mathrm{d}}^{\mathrm{UL}}=\frac{P_{d} G_{d d}}{N_{0}+I+P_{c} G_{c d}}$

where $P_{d}$ is the transmission power of the D2D user, $G_{d d}$ is the channel gain between the users of the D2D pair, $P_{c}$ is the transmission power of the cellular user, $G_{c d}$ is the channel gain between the cellular user and the D2D pair, $N_{0}$ is the noise in the system, and $I$ represents the interference from the neighboring cells. 
Similarly, while an eNodeB receives signal from the cellular user in uplink, it also receives interference from the uplink transmission of a D2D user using the same resources. Thus, the SINR at the eNodeB can be expressed as

$\gamma_{e N B}^{C}=\frac{P_{C} G_{C B}}{N_{0}+I+P_{d} G_{d B}}$

where $P_{C}$ is the transmission power of the cellular user, $G_{C B}$ is the channel gain between the eNodeB and the cellular user, $P_{d}$ is the transmission power of the D2D user, $G_{d B}$ is the channel gain between the eNodeB and the D2D pair, $N_{0}$ is the noise in the system, and $I$ represents the interference from the neighboring cells.

The SINR $\gamma_{\mathrm{d}}^{\mathrm{UL}}$ and $\gamma_{e N B}^{C}$, as determined from (8) and (9), must be enough high and this can be considered the primary radio condition that needs to be satisfied when a D2D communication is set up.

\section{PROPOSED METHOD FOR THE ESTIMATION OF THE FEASIBILITY OF SETTING UP A D2D COMMUNICATION}

It should be ensured that the radio conditions are satisfied prior to setting up D2D communication. In underlay inband mode, this requires that the interference is enough limited to cause enough high SINR. In this section, we propose that the feasibility of setting up a D2D communication can be estimated depending on how easily a cellular user can be found, which can share radio resources with the D2D pair while ensuring sufficient SINR.

To explain the proposed method of estimation, we consider a typical situation, in which the D2D pair is located at a large distance from the eNodeB and the distance between the two D2D users in the pair is sufficiently small. With the underlay inband implementation, the interference on the radio resources shared by both cellular user and D2D users must be kept below a certain level, in order to maintain sufficient SINR. This requires the cellular user to be located within a certain area of the cell. Assuming that the eNodeB is updated with the location information of most users in the cell, some of these users, ensuring sufficient SINR, can be preferably selected as cellular users, sharing radio resource with the D2D users. Such users are called suitable cellular users in this paper. The size of the area in the cell, with the possible existence of suitable cellular users, can be an indication of how easily they can be selected. We propose that the estimation of this size of the area can be considered as an indirect way to estimate the feasibility of setting up a D2D communication in a cell.

We present a simple method to quickly estimate the aforementioned feasibility. For this purpose, an estimation of SINR is used to determine whether or not the interference is enough limited due to the proper location of the cellular user. In other words, an area within the cell for the location of the cellular user, permitting enough high SINR, $\gamma_{\mathrm{d}}^{\mathrm{UL}}$ and $\gamma_{e N B}^{C}$, can first be determined and the cellular users, located within that area can be regarded suitable. According to the proposal, a computation of the size of the area for suitable cellular users, ensuring satisfactory SINR levels, compared to the whole cell area, can be performed in the real network, and this relative size can be used to estimate the feasibility of setting up a D2D communication. This proposed method of estimation is novel but simple, allowing easy and quick computation for the network. It may be noted that the necessary level of SINR depends on the scenario.

Typically, the D2D users, located close to each other, communicate between them using very low power transmission, and thus, $P_{d}$ and $G_{d B}$ take on very low values. Consequently, the SINR at the eNodeB, $\gamma_{e N B}^{C}$ takes on significantly higher values, compared to the SINR of the D2D user, $\gamma_{\mathrm{d}}^{\mathrm{UL}}$. Therefore, it is $\gamma_{\mathrm{d}}^{\mathrm{UL}}$ and not $\gamma_{e N B}^{C}$, which can be considered the limiting factor in SINR considerations. Thus, in the propose method, to estimate the feasibility of setting up D2D communication, the computation of the size of the area for suitable cellular users, needs to focus on ensuring a satisfactory level of $\gamma_{d}^{U L}$. 
For the purpose of illustration, we use the system model, presented in Section 4. Here, we assume a cell edge location for the D2D user. In this case, $G_{d d}$ can be calculated using (6), in which, it is denoted as $G_{u v}$. While using (6), $d_{u v}$ may be considered a fixed distance for simplicity. $G_{c d}, G_{c B}$, and $G_{d B}$ can be calculated using (4) and (5). While using (5), the distance $d$ for all different cases, may be determined using (1) - (3) for simplicity. In the case of $G_{c d}$, the distance $d$ is given by $a$. In the case of $G_{c B}$, the distance $d$ is given by $b$. In the case of $G_{d B}$, the distance $d$ is given by $m+n . P_{c}$ can be calculated using (7). $P_{d}$ may be calculated allowing certain received power at the receiving D2D user for the particular $G_{d d}$.

\section{SIMULATION}

A simulation of the proposed method for the estimation of the feasibility of setting up a D2D communication has been performed and the results are presented in this section. The system model, presented in Section 4, was used for the computation using the parameter values shown in Table 1. To determine the area for suitable cellular users in a cell, a polar plot has been used for a specific range of values of SINR. The position of the D2D pair is held fixed at the cell edge across the cell radius. The position of the cellular UE is varied with two-dimensionally in the cell, by varying the values of $m, n$, and $l$ with small granularities. As the cellular UE changes its position, the SINR and hence, the data rate experience of the UEs will vary. Other distances, $a$ and $b$, were calculated using (1) - (3). For simplicity, instead of using (7), the transmit power of the cellular UE, $P_{c}$ was calculated using an approximation that the uplink power control would keep the received power at eNodeB constant. Thus, as the position of the cellular UE varied, $P_{c}$ was adjusted such that the received power at eNodeB, $P_{C} G_{C B}$ was held constant at some particular value. This required calculating $G_{C B}$ for all positions of the cellular UE and (4) and (5) were used in this calculation. Using these values of $P_{c}$, the SINR at the D2D user $\gamma_{\mathrm{d}}^{\mathrm{UL}}$ was calculated using (8). In this case, the received power at the UE in D2D pair, $P_{d} G_{d d}$ was considered to have a fixed value, for simplicity. The calculation for $G_{c d}$, in (8), is performed using (4) and (5) for all positions of the cellular UE. Similarly, the SINR at the eNodeB, $\gamma_{e N B}^{C}$ was calculated using (9). $X_{\alpha}$ was set to 0 and $\left|H_{k}\right|^{2}$ was set to 1 in the use of (4), for simplicity.

In the simulation results, the SINR at the eNodeB, $\gamma_{e N B}^{C}$ was found to be far higher compared to the SINR of the D2D user, $\gamma_{\mathbf{d}}^{\mathrm{UL}}$. For example, when the cellular user was located at $141 \mathrm{~m}$ distance from the eNodeB across the cell radius, $\gamma_{\boldsymbol{e} N \boldsymbol{B}}^{\boldsymbol{C}}$ was found $12 \mathrm{~dB}$, whereas $\gamma_{\mathbf{d}}^{\mathrm{UL}}$ was found $-3 \mathrm{~dB}$. Therefore, $\boldsymbol{\gamma}_{\mathbf{d}}^{\mathrm{UL}}$ was set as the limiting factor. Figure 5 depicts how the SINR of D2D users, $\boldsymbol{\gamma}_{\mathbf{d}}^{\mathrm{UL}}$ decreases as the distance of the cellular user from eNodeB increases across the cell radius.

Considering various locations for the cellular user, it was checked whether $\gamma_{d}^{\mathrm{UL}}$ took satisfactory values or not. However, the satisfactory level of SINR $\gamma_{d}^{\mathrm{UL}}$ can vary depending on the scenario. So, the area for the suitable cellular user within the cell, permitting different particular ranges of $\gamma_{\mathrm{d}}^{\mathrm{UL}}$, was determined. A MATLAB program was developed for both the determination and marking of this area. The polar plot, in MATLAB, was used technically for the purpose of marking in red. Figure 6-8 show the red marked areas in the cell, indicating the location of the suitable cellular users, for various specific ranges of values of SINR $\gamma_{d}^{\mathrm{UL}}$. In these figures, the area between $60^{\circ}$ and $300^{\circ}$ indicates the whole cell area. According to the proposed method, the larger the red marked area compared to the whole the cell area, the greater is the feasibility of setting up the D2D communication. 
Table I. Simulation Assumptions

\begin{tabular}{|c|c|}
\hline Parameter & Value \\
\hline Cell shape & $\begin{array}{c}\text { With three equally spaced cells under } \\
\text { an eNodeB of circular coverage, } \\
\text { each cell has } 120^{0} \text { angular spacing }\end{array}$ \\
\hline Cell radius, $m+n$ & Uniform \\
\hline $\begin{array}{c}\text { Distribution of cellular users in the } \\
\text { cell }\end{array}$ & At the cell edge, across the cell radius \\
\hline Position of the D2D pair & $2000 \mathrm{MHz}$ \\
\hline Carrier frequency ( $\mathrm{f}_{\mathrm{c}}$ ) & $25 \mathrm{~m}$ \\
\hline eNodeB antenna height $\left(\mathrm{h}_{\mathrm{b}}\right)$ & $3.98107 \times 10^{-9} \mathrm{~mW}$ \\
\hline $\begin{array}{c}\text { Noise in the system (receiver's } \\
\text { thermal noise), } \mathrm{N}_{0}\end{array}$ & $-80 \mathrm{dBm}$ \\
\hline $\begin{array}{c}\text { Total Interference from the } \\
\text { neighboring cells at eNodeB, } I\end{array}$ & $-80 \mathrm{dBm}$ \\
\hline $\begin{array}{c}\text { Total Interference received from the } \\
\text { neighboring cells at UE in D2D } \\
\text { pair, } I\end{array}$ & 1 \\
\hline $\begin{array}{c}\text { Normalized constant, } \mathrm{K}_{\mathrm{uv}} \\
\text { Received power at eNodeB from } \\
\text { cellular user (fixed) }\end{array}$ & $-60 \mathrm{dBm}$ \\
\hline $\begin{array}{c}\text { Received power at UE of D2D pair } \\
\text { (fixed) }\end{array}$ & $-60 \mathrm{dBm}$ \\
\hline
\end{tabular}

In Figure 6-8, the ratio between the size of the red marked area, permitted location for suitable cellular users, and the whole cell area, is considered the feasibility of setting up D2D communications. In Figure 9, a probability density function (PDF) is thus plotted for different ranges of SINR $\gamma_{d}^{\mathrm{UL}}$. As demonstrated, when considered a specific range, the probability increases gradually with the level of the range up to a maximum level, and thereafter, decreases gradually. Here, with a $5 \mathrm{~dB}$ wide range of value, between $-5 \mathrm{~dB}$ and $0 \mathrm{~dB}$, the probability takes on the maximum. This is also reflected in Figure 6, as the plot for $-5 \mathrm{~dB}<\mathrm{SINR}<0 \mathrm{~dB}$ has a larger red marked area compared to other plots. In Figure 10 , a cumulative density function (CDF) is plotted. As expected, as the permissible SINR value can be made lower, the feasibility of setting up D2D communication increases.

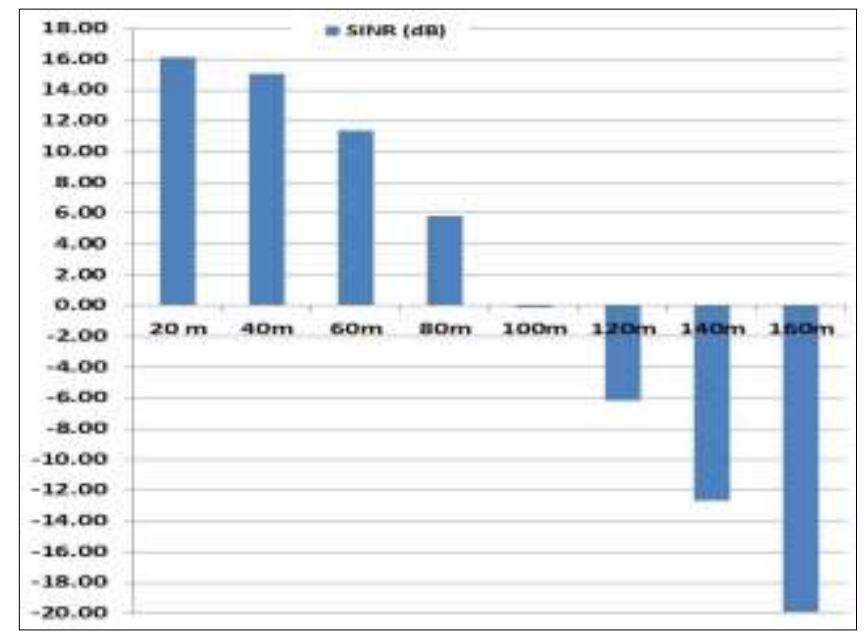

Fig. 5 SINR $\gamma_{\mathrm{d}}^{\mathrm{UL}}$ vs. distance of the cellular user from eNodeB 


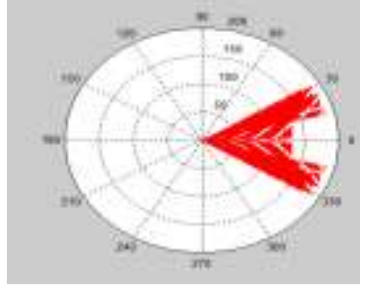

(a) $-10 \mathrm{~dB}<\mathrm{SINR}<-5 \mathrm{~dB}$

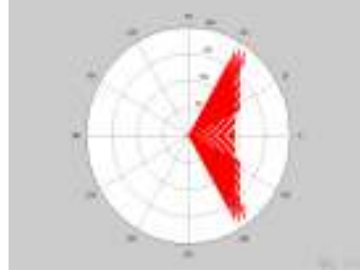

(c) $0 \mathrm{~dB}<\mathrm{SINR}<5 \mathrm{~dB}$

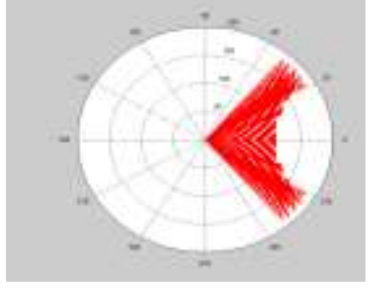

(b) $-5 \mathrm{~dB}<\mathrm{SINR}<0 \mathrm{~dB}$

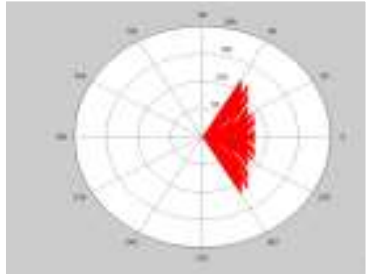

(d) $5 \mathrm{~dB}<\mathrm{SINR}<10 \mathrm{~dB}$

Fig. 6 Red marked areas for suitable cellular users, allowing specific ranges of SINR, $\gamma_{\mathrm{d}}^{\mathrm{UL}}$

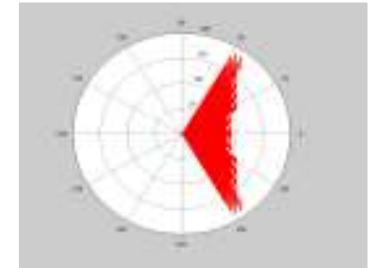

(a) SINR greater than $0 \mathrm{~dB}$

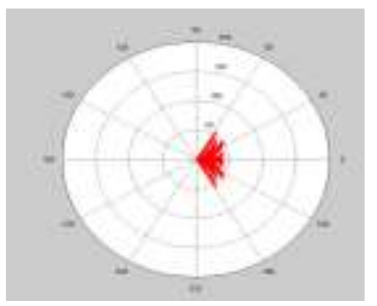

(c) SINR greater than $15 \mathrm{~dB}$

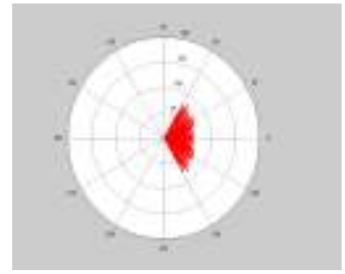

(b) SINR greater than $10 \mathrm{~dB}$

Fig. 7 Red marked areas for suitable cellular users, allowing specific ranges of SINR, $\gamma_{\mathbf{d}}^{\mathbf{U}}$ 


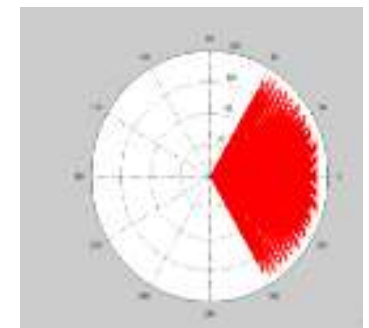

(a) SINR greater than $-40 \mathrm{~dB}$

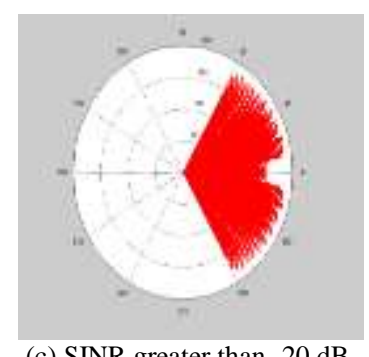

(c) SINR greater than $-20 \mathrm{~dB}$

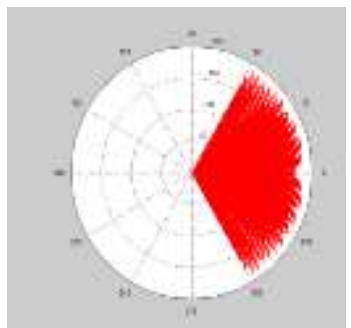

(b) SINR greater than $-30 \mathrm{~dB}$

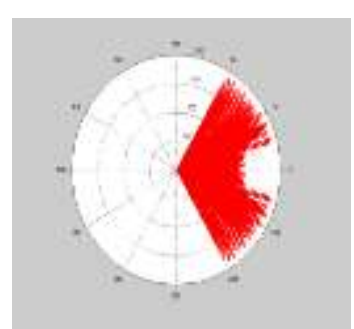

(d) SINR greater than $-10 \mathrm{~dB}$

Fig. 8 Red marked areas for suitable cellular users, allowing specific ranges of SINR, $\gamma_{\mathrm{d}}^{\mathrm{UL}}$

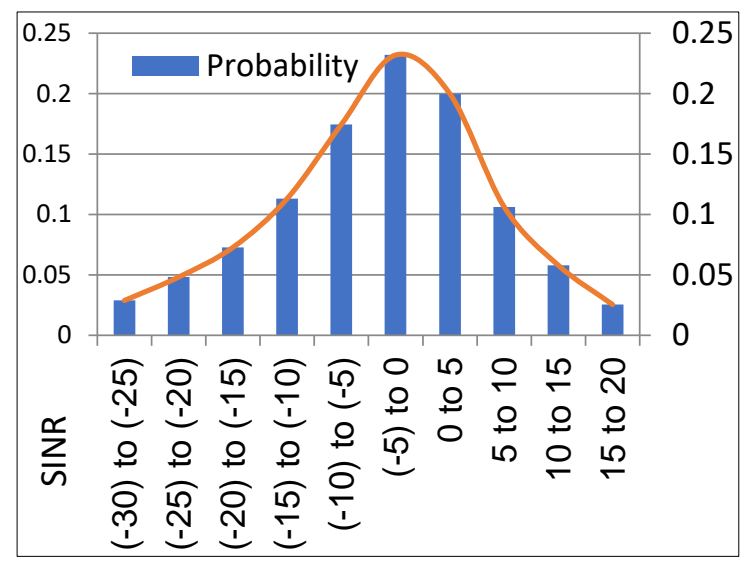

Fig. 9 Probability density function (PDF) of setting up D2D communication for different ranges of SINR $\boldsymbol{\gamma}_{\mathbf{d}}^{\mathrm{UL}}$

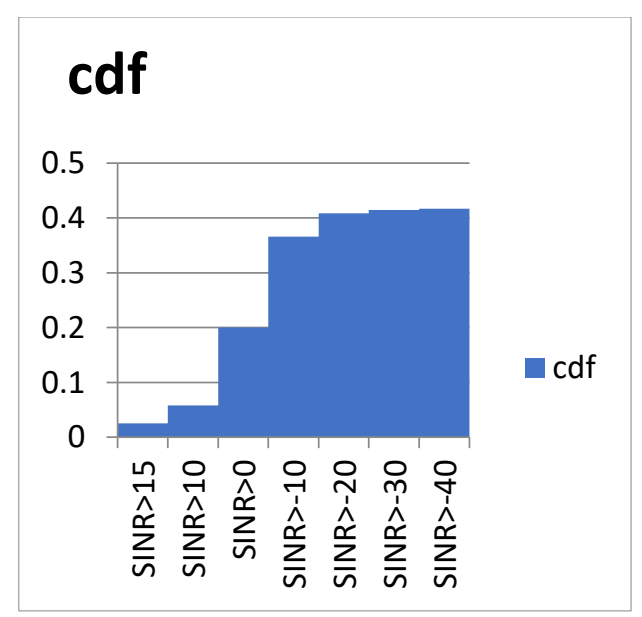

Fig. 10 Cumulative density function (CDF) of setting up D2D communication for different ranges of SINR $\boldsymbol{\gamma}_{\mathbf{d}}^{\mathbf{U L}}$ 


\section{CONCLUSION}

D2D communication offers several advantages, which are not available in conventional cellular communication. However, the interference management is crucial while setting up a D2D communication. A simple method is proposed for determining the feasibility of D2D communication in a cell, considering the permissible level of interference experienced by the users. The method is presented considering only the use of uplink resources for D2D communication. However, the method could be similarly derived for the case of the use of downlink resources. A sample computation has been performed using the proposed method, which demonstrates how the feasibility increases as the permissible level of interference can be sacrificed. Since the proposed method is simple and quick, it can be easily employed in the live network.

\section{REFERENCES}

[1] G. Fodor and N. Reider, "A Distributed Power Control and Mode Selection Algorithm for D2D Communications", EURASIP Journal on Wireless Communications and Networking (2012). DOI:10.1186/1687-1499-2012-266.

[2] Runhua Chen and J.G. Andrews, "Uplink Power Control in Multi-Cell Spatial Multiplexing Wireless Systems", IEEE Transactions on Wireless Communication, vol. 6, no. 7, (2007), pp. 2700 - 2711. DOI: 10.1109/TWC.2007.051007.

[3] C. Chien, Y. Chen and H. Hsieh, "Exploiting Spatial Reuse Gain through Joint Mode Selection and Resource Allocation for Underlay Device-to-Device Communications." 15th International Symposium on Wireless Multimedia Communications (WPMC), Taipei, Taiwan, (24-27 September 2012), pp. 80-84. https://ieeexplore.ieee.org/document/6398841.

[4] J. Du, W. Zhu, J. Xu, Z. Li, and H. Wang, "A compressed HARQ feedback for device-to-device multicast communications." IEEE Vehicular Technology Conference (VTC Fall), Quebec City, QC, Canada, (3-6 September 2012), pp. 1-5. DOI: 10.1109/VTCFall.2012.6399309.

[5] L. Lei, Z. Zhong, C. Lin, and X. Shen, "Operator controlled device-to-device communications in LTEadvanced networks", IEEE Wireless Communications, vol. 19, no. 3, (2012), pp. 96-104. DOI: 10.1109/MWC.2012.6231164.

[6] K. Doppler, M. Rinne, C. Wijting, C. Ribeiro, and K. Hugl, "Device-to-device communication as an underlay to LTE-advanced networks", IEEE Communications Magazine, vol. 47, no. 12, (2009), pp. 4249. DOI: 10.1109/MCOM.2009.5350367.

[7] N. Golrezaei, Parisa Mansourifard, A. F. Molisch, and A. G. Dimakis, "Base-station assisted device-todevice communications for high-throughput wireless video networks", IEEE Transactions on Wireless Communications, Vol. 13, no. 7, (2012), pp. 3665-3676. DOI: 10.1109/TWC.2014.2316817.

[8] N. Golrezaei, A. G. Dimakis, and A. F. Molisch, "Device-to-device collaboration through distributed storage", 2012 IEEE Global Communications Conference (GLOBECOM), Anaheim, CA, USA, (3-7 December 2012), pp. 2397-2402. DOI: 10.1109/GLOCOM.2012.6503475.

[9] J. C. Li, M. Lei, and F. Gao, "Device-to-Device (D2D) communication in MU-MIMO cellular networks", 2012 IEEE Global Communications Conference (GLOBECOM), Anaheim, CA, USA, (3-7 December 2012), pp. 3583-3587. DOI: 10.1109/GLOCOM.2012.6503671.

[10] N. K. Pratas and P. Popovski, "Low-rate machine-type communication via wireless Device-to-Device (D2D) links", arXiv preprint (2013). https://arxiv.org/abs/1305.6783.

[11] Arash Asadi, Qing Wang, and Vincenzo Mancuso, "A Survey on Device-to-Device Communication in cellular network", IEEE Communications Surveys \& Tutorials, vol. 16, no. 4, (2014), pp. 1801-1819. DOI: 10.1109/COMST.2014.2319555.

[12] Haichuan Ding, Ma Shaodan, and Xing Chengwen, "Feasible D2D communication distance in D2Denabled cellular networks", In 2014 IEEE International Conference on Communication Systems, (2014) pp. $1-5$.

[13] A. Osseiran, K. Doppler, C. Ribeiro, M. Xiao, M. Skoglund, and J. Manssour, “Advances in device-todevice communications and Network coding for IMT-Advanced", 2nd International Conference on Wireless Communication, Vehicular Technology, Information Theory and Aerospace \& Electronic Systems Technology (Wireless VITAE), Chennai, India, (28 February-3 March 2011). DOI: 10.1109/WIRELESSVITAE.2011.5940847.

[14] C. Xu, L. Song, Z. Han, Q. Zhao, X. Wang, and B. Jiao, "Interference aware resource allocation for device-to-device communications as an underlay using sequential second price auction”, 2012 IEEE International Conference on Communications (ICC), Ottawa, ON, Canada, 10-15, (June 2012), pp. 445449. DOI: 10.1109/ICC.2012.6364509.

[15] S. Xu, H. Wang, T. Chen, Q. Huang, and T. Peng, "Effective interference cancellation scheme for deviceto-device communication underlaying cellular networks", IEEE 72nd Vehicular Technology Conference, Canada, (September 2010), pp. 1-5. DOI: 10.1109/VETECF.2010.5594601. 
[16] W. Xu, L. Liang, H. Zhang, S. Jin, J. C. Li, and M. Lei, "Performance enhanced transmission in deviceto-device communications: Beamforming or interference cancellation", 2012 IEEE Global Communications Conference (GLOBECOM), Anaheim, CA, USA, (3-7 December 2012), pp. 42964301. DOI: 10.1109/GLOCOM.2012.6503793.

[17] B. Zhou, H. Hu, S.-Q. Huang, and H.-H. Chen, "Intracluster device-to-device relay algorithm with optimal resource utilization", IEEE Transactions on Vehicular Technology, vol. 62, no. 5, (2013), pp. 2315-2326. DOI: 10.1109/TVT.2012.2237557.

[18] X. Bao, U. Lee, I. Rimac, and R. R. Choudhury, "Data Spotting: offloading cellular traffic via managed device-to-device data transfer at data spots", ACM SIGMOBILE Mobile Computing and Communications Review, vol. 14, no. 3, (2010), pp. 37-39. DOI:10.1145/1923641.1923655.

[19] A. Asadi and V. Mancuso, "Energy efficient opportunistic uplink packet forwarding in hybrid wireless networks", Proceedings of the fourth international conference on Future energy systems, Berkeley, California, USA, (21 - 24 May, 2013), pp. 261-262. DOI: 10.1145/2487166.2487197.

[20] A. Asadi and V. Mancuso, "On the compound impact of opportunistic scheduling and D2D communications in cellular networks", Proceedings of the 16th ACM international conference on Modeling, analysis \& simulation of wireless and mobile systems, Barcelona, Spain, (3-8 November, 2013), pp. 279-288. DOI: 10.1145/2507924.2507929.

[21] M. Ji, G. Caire, and A. F. Molisch, "Wireless device-to-device caching networks: Basic principles and system performance." IEEE Journal on Selected Areas in Communications, vol. 34, no. 1, (2016), pp. 176-189. DOI: 10.1109/JSAC.2015.2452672.

[22] M. Zulhasnine, C. Huang, and A. Srinivasan, "Penalty function method for peer selection over wireless mesh network", 2010 IEEE 72nd Vehicular Technology Conference, Ottawa, ON, Canada, (6-9 September, 2010), pp. DOI: 10.1109/VETECF.2010.5594219.

[23] Mohammad T. Kawser, "LTE Air Interface Protocols", Boston, USA, Artech House, (2011). ISBN: 9781-60807-201-9. 